\title{
La práctica del Tai Chi previene las caídas en el Anciano Institucionalizado: Un Ensayo Clínico
}

\author{
Beatriz Valero Serrano ${ }^{\mathrm{a}}$, Pablo Franquelo Morales ${ }^{\mathrm{a}}$, Félix González Martíneza, \\ Javier de León Belmara, José Luis Quijada Rodríguez ${ }^{\mathrm{b}}$
}

\begin{abstract}
a Servicio de Urgencias, Hospital Virgen de la Luz, Cuenca.

b Servicio de Traumatología, Hospital Virgen de la Luz, Cuenca.

\section{Correspondencia:}

Beatriz Valero Serrano, Servicio de Urgencias, Hospital Virgen de la Luz, C/ Hermandad de Donantes de Sangre $n^{\circ} 2$, 16002 - Cuenca, España. E-mail:

bvaleroserrano@gmail.com.

Recibido el 15 de octubre de 2009.
\end{abstract}

Aceptado para su publicación el 10 de enero de 2010.

\section{RESUMEN}

Objetivo. Determinar si la práctica de dos horas de Tai Chi semanal durante 9 meses disminuye el riesgo de caídas en ancianos de edad igual o superior a 65 años.

Diseño. Ensayo clínico con aleatorización por grupos sin cegamiento.

Emplazamiento. Dos residencias de ancianos de la ciudad de Cuenca.

Participantes. Un total de 94 ancianos institucionalizados, de entre 65 y 90 años. Grupo control de 43 y grupo de intervención de 51 practicantes de Tai Chi.

Mediciones principales. Mediante entrevista semanal, realizada por uno de los investigadores, se recogieron como eventos principales el número de caídas, número de fracturas, número de tropiezos y pérdidas de equilibrio.

Resultados. La incidencia de caídas en el grupo de Tai Chi fue del $13,7 \%$ y en el grupo control del 32,5\% ( $p<0,03$ ); Razón de Riesgo $[R R]=0,42$, lo que supone una Reducción de Riesgo (RAR) del 18,8\%. Como resultado de estas caídas, 5 ancianos de los controles $(11,6 \%)$ sufrieron fracturas óseas y ninguna en el grupo de Tai Chi $(p<0,02)$. Encontramos diferencias estadísticamente significativas en cuanto al número de tropiezos, 16 en el grupo de Tai Chi $(31,3 \%)$ y 49 en los controles $(113,9 \%)(p<0,04$; RR $=0,28$; RAR $=0,826)$. Igualmente las diferencias en el número de pérdidas de equilibrio fue significativa, 12 en el grupo de Tai Chi $(23,5 \%)$ y 37 en el grupo control $(86 \%)(p<0,02 ; R R=0,27$; RAR = 0,625 ).

Conclusiones. La práctica regular de Tai Chi probablemente disminuye el número de caídas, la incidencia de fracturas, el número de tropiezos y las pérdidas de equilibrio en pacientes ancianos institucionalizados.

Palabras clave. Tai Chi, Accidentes por Caídas, Anciano.

\section{ABSTRACT}

The practice of Thai-Chi prevents falls in the institutionalized elderly: a clinical trial Objective. To determine whether practicing Thai Chi for 2 hours a week for 9 months decreases the risk of falls in elderly people aged 65 years or over living in residential care homes.

Design. Randomised, non-blind clinical trial with two groups.

Setting. Two residential care homes in the city of Cuenca.

Participants. A total of 94 institutionalized elderly persons between 65 and 90 years. The control group comprised 43 persons and the Thai Chi group 51 persons.

Main measurements. The main measurements were number of falls and their severity, the number of stumbles and losses of balance determined by a weekly interview with one of the investigators.

Results. The incidence of falls was $13.7 \%$ in the Tai Chi group and $32.5 \%$ in the control group. ( $p<0.03$ ); Relative Risk, $(R R)=0.42$, representing a risk reduction of $18.8 \%$. Five elderly persons in the control group (11.6\%) and none in the Thai Chi Group $(p<0.02)$ suffered bone fractures as result of the falls. We found significant differences in the number of stumbles, 16 in the Thai Chi group $(31.3 \%)$ and 49 in control group $(113.9 \%)(p<0.04$; $\mathrm{RR}=0.28$; RAR $=0.826)$. Likewise, the differences in the number of losses of balance was significant, 12 in the Thai Chi group (23.5\%) and 37 in the control group (86\%) $(p<0.02$; $\mathrm{RR}=0.27$; RAR = 0.625).

Conclusions. Regular Thai Chi practice may reduce the number of falls, the incidence of bone fractures, the number of stumbles and losses of balance in elderly people living in residential care homes.

Key words. Tai Ji, Accidental Falls, Aged. 


\section{INTRODUCCIÓN}

Las caídas en los ancianos constituyen un problema de salud pública, siendo una de las principales causas de lesión, incapacidad y muerte, además de un indicador para la detección del anciano frágil (personas mayores que por su situación física, psíquica, funcional o social se encuentran en un equilibrio inestable, presentando una gran probabilidad de sufrir dependencia o institucionalización).

Se estima que el $30 \%$ de las personas de más de 65 años sufre al menos una caída al año ${ }^{1-2}$, siendo este porcentaje mayor en los ancianos institucionalizados, hasta un $50 \%{ }^{2-5}$. En la mitad de los casos las caídas son recurrentes ${ }^{2,5,6}$ y al menos el $20 \%$ requiere asistencia médica. La dependencia de otros y el miedo a caer de nuevo, son las principales consecuencias psicológicas. Así, en el "Síndrome Postcaída", que aparece en ancianos con caídas de repetición, el miedo a caerse de nuevo puede llegar a incapacitarles posteriormente para la marcha ${ }^{7,8,16}$.

Estudios observacionales han relacionado las caídas con alteraciones en la marcha, equilibrio y postura, alteraciones cognitivas, visuales, auditivas, disminución de la fuerza muscular, anormalidades articulares, el uso de cuatro o más medicamentos, síntomas depresivos, síncopes e hipotensión ortostática entre otros ${ }^{8,-10}$, mostrando un incremento de la probabilidad de caída a medida que se suman los factores de riesgo ${ }^{3}$.

Actualmente existe un interés creciente en evaluar intervenciones que disminuyan la incidencia de caídas $^{1}$, como el ejercicio físico, que ha demostrado beneficios para la salud física, psicológica y psicosocial ${ }^{4,5,11}$. El Tai Chi ha sido practicado desde hace siglos en las culturas orientales como ejercicio físico para mejorar el equilibrio y la percepción del esquema corporal, y recientemente se ha incrementado su práctica en la población anciana occidental. Actualmente, existen estudios que demuestran una evidente mejora en el equilibrio, estabilidad postural, función cardiorrespiratoria, presión sanguínea, capacidad aeróbica, fuerza, flexibilidad y un mejor estado físico, mental y psico-social en los sujetos que lo practican ${ }^{12-30}$.

Los ancianos institucionalizados presentan un mayor riesgo de caída ${ }^{30}$, sin embargo, la mayoría de los estudios diseñados para prevenir las caídas han tomado como muestras ancianos que viven en la comunidad y que no cumplen criterios de fragilidad. Nuestro objetivo es determinar si la práctica regular de Tai-Chi en ancianos institucionalizados provoca una disminución en el número de caídas o bien en la gravedad de éstas.

\section{MATERIAL Y MÉTODO}

El ensayo clínico se desarrolló con 102 ancianos institucionalizados en dos residencias de ancianos en la ciudad de Cuenca (figura 1). Ocho sujetos no cumplieron los criterios de inclusión (tabla 1). Se realizó una aleatorización por grupos, asignando de forma aleatoria el grupo de intervención (Tai Chi) a una de las residencias y el control a otra. No se realizó aleatorización individual. El tamaño muestral fue calculado teniendo en cuenta un error alfa de 0,05 , un error beta de 0,20 y una diferencia del tamaño del efecto en la incidencia de caídas del $30 \%$.

La inclusión en el estudio fue voluntaria, obteniendo previamente por escrito el consentimiento informado. El proyecto fue aprobado por el Comité Ético de Investigación Clínica del Área de Salud de Cuenca.

No se realizó ningún tipo de enmascaramiento. Tampoco se realizó evaluación ciega por terceros, ya que los resultados fueron recogidos por uno de los investigadores, el cual conocía el grupo de pertenencia de los sujetos.

Con el fin de evaluar la homogeneidad de los grupos, además de las variables sociodemográficas

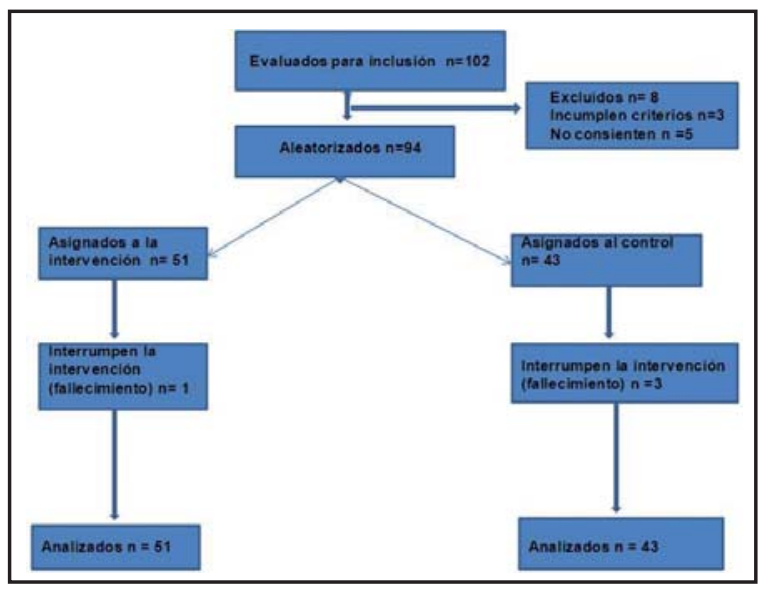

Figura 1. Diagrama de flujo de participantes

\begin{tabular}{l|l}
\hline Criterios de inclusión & Criterios de exclusión \\
\hline Ser mayores de 65 años & $\begin{array}{l}\text { Padecer signos o síntomas de } \\
\text { inestabilidad en enfermedades } \\
\text { cardiopulmonares, neurológiocas o } \\
\text { o con ayuda de bastón/andador tipo de patología médica }\end{array}$ \\
No cumplir criterios de exclusión & $\begin{array}{l}\text { Deterioro cognitivo definido como } \\
\text { puntuación mayor de 5 en } \\
\text { el cuestionario abreviado de Pfeiffer } \\
\text { Contraindicaciones para realizar } \\
\text { ejercicio físico } \\
\text { Sufrir procesos terminales } \\
\text { La falta de asistencia a la clase durante } \\
8 \text { semanas consecutivas }\end{array}$ \\
\hline
\end{tabular}

Tabla 1. Criterios de inclusión y exclusión. 
se recogieron mediante diversos cuestionarios la situación funcional, cognitiva y afectiva de los participantes, mediante el Îndice de Barthel, el cuestionario abreviado de Pfeiffer y la escala de depresión de Yesavage. Con el test de Tinetti se evaluó el equilibrio estático y dinámico. También se registró el índice Karnofsky. Se estimó el miedo a la caída a través de una encuesta (miedo nulo, moderado, alto o muy alto) y se registraron las cifras de TA con medidas esfingomanométricas estandarizadas, la frecuencia cardiaca (FC), el índice de masa corporal (IMC) y la agudeza visual (AV). Se consignó si habían sufrido alguna caída en el año anterior al estudio. También se recogieron los antecedentes médico-quirúrgicos y el tratamiento farmacológico habitual.

Durante el transcurso de 9 meses, en el grupo de intervención los instructores de Tai Chi impartieron clases de una hora de duración, dos veces por semana. En cada sesión se realizaron una serie de ejercicios de calentamiento de aproximadamente 15 minutos y posteriormente se practicaron los movimientos registrados en la tabla de Tai Chi. En el grupo control se proporcionó a los ancianos recomendaciones para la prevención de caídas, consejos dietéticos, nutricionales e higiene para la salud, pero no se les facilitaron instrucciones formales sobre la práctica de ejercicio.

Durante 9 meses, con frecuencia semanal, el investigador principal recogió mediante entrevista personal el número de caídas, la presentación de fracturas, el número de tropiezos y las pérdidas de equilibrio, en ambos grupos. Definimos caída como "el cambio brusco no intencionado desde postura en bipedestación hasta el suelo o plano inferior, con o sin pérdida de conciencia, relatado por el afectado o por un testigo". Se consideró tropiezo como "dar con los pies en algún estorbo que lo situara en riesgo de caída" y desequilibrios las "pérdidas momentáneas de estabilidad en la postura desde la bipedestación, sin llegar a caer al suelo".

Se realizó un análisis por intención de tratar, asignando a los sujetos perdidos los valores de la última observación disponible (locf). El estudio estadístico se realizó mediante el test t de Student para comparar las medias de dos muestras independientes y la $U$ de Mann-Whitney para valorar la asociación estadística entre variables cuantitativas. Para determinar la asociación estadística entre las variables cualitativas dicotómicas se utilizó el test de chi-cuadrado. La fuerza de asociación se determinó mediante la razón de riesgo (RR), la reducción del riesgo (RAR) y el número de sujetos necesarios a tratar para evitar un evento negativo (NNT). El análisis estadístico se realizó con el programa SPSS para Windows en su versión 14.0.

\section{RESULTADOS}

Concurrió homogeneidad en ambos grupos respecto a las variables consideradas, excepto en la incidencia de caídas en el año previo al estudio: 16 $(31,4 \%)$ en el grupo de Tai Chi y $5(11,6 \%)$ en el grupo control $(p<0.02)$ (tabla 2$)$.

\begin{tabular}{|c|c|c|c|}
\hline & $\begin{array}{l}\text { GRUPO TAI CHI } \\
\mathrm{N}=51\end{array}$ & $\begin{array}{l}\text { GRUPO CONTROL } \\
N=43\end{array}$ & \\
\hline Variables Cuantitativas & Media $\pm \mathrm{DE}$ & Medi \pm DE & DNS \\
\hline Tiempo con ayuda para caminar & $4,25 \pm 4,66$ & $3,95 \pm 2,86$ & DNS \\
\hline Edad Media & $81,78 \pm 5,4$ & $81,07 \pm 7,5$ & DNS \\
\hline Peso & $66,42 \pm 10,91$ & $69 \pm 9,7$ & DNS \\
\hline Talla & $1,55 \pm 0,06$ & $1,60 \pm 0,15$ & DNS \\
\hline IMC & $27,55 \pm 4,82$ & $28,65 \pm 4,98$ & DNS \\
\hline TAS & $139,86 \pm 21,06$ & $136,56 \pm 19,78$ & DNS \\
\hline TAD & $79,48 \pm 12,54$ & $77,90 \pm 15,63$ & DNS \\
\hline FC & $72,66 \pm 8,59$ & $79,60 \pm 8,99$ & DNS \\
\hline AV OD & $0,57 \pm 0,30$ & $0,59 \pm 0,15$ & DNS \\
\hline AV OI & $0,56 \pm 0,28$ & $0,57 \pm 0,30$ & DNS \\
\hline Índice Karnofski & $95 \pm 7,5$ & $95 \pm 9,2$ & DNS \\
\hline Índice Barthel & $60 \pm 0$ & $60 \pm 0$ & DNS \\
\hline Índice Pfeiffer & $2,30 \pm 1,98$ & $2,10 \pm 2$ & DNS \\
\hline Índice Yesavage & $2,90 \pm 2,29$ & $2,80 \pm 3,56$ & DNS \\
\hline Índice Tinetti & $88,62 \pm 11,51$ & $87,21 \pm 9,11$ & DNS \\
\hline Variables Cualitativas & N (\%) & N (\%) & \\
\hline Sexo masculino & $19(37 \%)$ & $22(51,2 \%)$ & DNS \\
\hline Sexo femenino & $32(63 \%)$ & $21(48,8 \%)$ & \\
\hline Pacientes con caídas previas & $16(31,4 \%)$ & $5(11,6 \%)$ & $p<0,02$ \\
\hline \multicolumn{4}{|l|}{ Miedo de Caída } \\
\hline - Nulo & $6(11,85)$ & $7(16,3 \%)$ & \\
\hline - Leve & $11(21,6 \%)$ & $12(27,9 \%)$ & \\
\hline - Moderado & $18(35,3 \%)$ & $10(23,3 \%)$ & DNS \\
\hline - Alto & $10(11,8 \%)$ & $9(20,9 \%)$ & \\
\hline - Muy alto & $6(54,5 \%)$ & $5(11,6 \%)$ & \\
\hline
\end{tabular}

Tabla 2. Características basales. DNS: Diferencia no significativa. 
Tras los nueve meses de seguimiento, se encontró una disminución significativa de la incidencia de caídas $(p=0,03)$, fracturas $(p<0,02)$, tropiezos $(p$
$=0,04)$ y pérdidas de equilibrio $(p=0,02)$ en los pacientes el grupo de intervención (tabla 3 ).

\begin{tabular}{|c|c|c|c|c|c|c|}
\hline & GRUPO TAI CHI & GRUPO CONTROL & $\mathbf{P}$ & RR & RAR & NNT \\
\hline Caídas & $7 / 51(13,7 \%)$ & $14 / 43(32,5 \%)$ & $p<0,03$ & 0,42 & $18,8 \%$ & 5,3 \\
\hline Fracturas & $0 / 51(0 \%)$ & $5 / 51(11,6 \%)$ & $p<0,02$ & & & \\
\hline Tropiezos & $16 / 51(31,3 \%)$ & $49 / 43(113,9 \%)$ & $p<0,04$ & 0,28 & $82,6 \%$ & 1,2 \\
\hline Pérdidas equilibrio & $12 / 51(23,5 \%)$ & $37 / 43(86 \%)$ & $p<0,02$ & 0,27 & $62,5 \%$ & 1,6 \\
\hline
\end{tabular}

Tabla 3. Resultados.

Durante el periodo de estudio fallecieron 4 participantes, 1 en el grupo de Tai Chi y 3 en los controles, resultando no significativa la diferencia. No se produjo ningún efecto adverso durante el desarrollo del estudio.

\section{DISCUSIÓN}

Los resultados de este estudio demostraron que la práctica de Tai Chi en ancianos institucionalizados disminuye el riesgo de tropiezos, caídas y pérdidas de equilibrio de forma significativa.

En nuestra opinión, la reducción en la incidencia de estos eventos es de especial relevancia, dado que los desequilibrios y los tropiezos suponen un riesgo potencial de caídas. También encontramos que la gravedad de las caídas fue menor, ya que los ancianos del grupo Tai Chi no sufrieron ninguna fractura, mientras que el $11,6 \%$ de las caídas de los controles tuvieron como resultado alguna lesión ósea. Este hecho cobra especial importancia en lo que se refiere a la calidad de vida y la capacidad funcional de los ancianos.

Los grupos eran homogéneos en todos los parámetros evaluados al inicio del estudio, salvo un mayor porcentaje de caídas, en el año previo al estudio, en el grupo de Tai Chi con respecto al control, lo que podría evidenciar una mejoría física aún mayor en el grupo de Tai Chi si consideramos que las caídas previas son un factor de riesgo para nuevas caídas $^{13}$, aunque este hecho pudiera deberse también a la aleatorización por grupos.

En el ensayo clínico prospectivo randomizado Atlanta FICSIT (Frailty and Injuries: Cooperative Studies of Intervention Techniques) ${ }^{1}$, Wolf demostró que la práctica de 10 de los 108 movimientos de la tabla de Tai-Chi, en ancianos mayores de 70 años que vivían en la comunidad, durante un periodo de 15 semanas, suponía una reducción del riesgo de caídas con respecto a los controles del $47 \%$, con una RR de 0,5 (IC 95\%: 0,36-0,37). Sin embargo, no se encontraron diferencias estadísticamente significativas en un estudio posterior que trataba de determinar el efecto de la práctica de dos horas semanales de Tai Chi, durante 48 semanas, por ancianos que cumplían algunos criterios de fragilidad, en comparación con un grupo control que recibía clases de educación para la salud ${ }^{29}$. Los eventos de caídas eran recogidos a través de postales que los accidentados debían remitir a los investigadores. En cambio en nuestro estudio, con un seguimiento de 9 meses, las caídas fueron registrándose mediante entrevistas semanales con los ancianos, para evitar sesgos por fallos mnésicos o por desidia en los participantes. Wolf no encontró diferencias en cuanto a la gravedad de las caídas, a diferencia de lo hallado en nuestros resultados. Tampoco en estudios previos se han evaluado los tropiezos ni desequilibrios.

Por las características de nuestra intervención, no fue posible obviamente el enmascaramiento a doble ciego. Tampoco se realizó evaluación ciega por terceros debido a las limitaciones económicas y de recursos del estudio. No se realizó aleatorización individual, también debido a la limitación de recursos humanos y materiales.

La mejora en la función física producida por el Tai Chi sugiere la necesidad de considerar la extensión de esta práctica de ejercicio como estrategia para promover la autonomía de los ancianos. Desde un punto de vista de salud pública, el Tai Chi es una herramienta barata y eficaz en la reducción del riesgo de caída y minimiza el declinar físico de los ancianos, produciendo una clara mejora en su calidad de vida.

En conclusión, la práctica de Tai Chi probablemente es eficaz en la disminución de la incidencia de tropiezos, pérdidas de equilibrio, caídas y consecuencias de las mismas en los ancianos institucionalizados. Para afianzar esta conclusión sería importante realizar un ensayo clínico con aleatorización individual y evaluación ciega por terceros.

\section{AGRADECIMIENTOS}

Asociación de Tai Chi Taoísta de Cuenca. 


\section{BIBLIOGRAFÍA}

1. Gillespie LD, Gillespie WJ, Robertson MC, Lambs SE, Cummings RG, Rowe BH. Interventions for preventing falls in the older people living in the community. Cochrane database Syst Rev. 2009; 15 (2):CD007146.

2. Vu MQ, Weintrabu N, Rubenstein LZ. Falls in the nursing home: are they preventable? J Am Med Dir Assoc. 2004; 5:401-6.

3. Jensen J, Lundin-Olsson I, Nyberg L, Gustafson Y. Fall and injury prevention in older people living in residential care facilities. Ann Intern Med. 2002; 136:733-41.

4. Tinetti ME. Preventing falls in elderly persons. $\mathrm{N}$ Engl J Med. 2003; 348:42-49.

5. Province MA, Haddley EC, Hornbrook MC, Lipsitz LA, Miller JP, Mulrow CD, et al. The effects of exercise on falls in elderly patients. A preplanned meta-analysis of the FICSIT Trials. Frailty and Injuries: Cooperative Studies of Intervention Techniques. JAMA. 1995; 273:1341-7.

6. Schumway-Cook A, Silver IF, LeMier M, York S, Cummings P. Effectiveness of community-based multifactorial Intervention on fall risk factors in community-living older adults: a randomized, controlled trial. J Gerontol A Biol Sci Med Sci. 2007; 62:1420-7.

7. Murphy Jet Coll. The post-fall Syndrome. Gerontology. 1982; 28:265-70.

8. Parrilla-Ruiz FM, Vargas-Ortega DA, Cárdenas-Cruz DP, Martínez-Cabezas S. Factores de riesgo, precipitantes, etiología y consecuencias de las caídas en el anciano. Medicina de Familia. 2004; 1:31-34.

9. Tinetti ME, Baker DI, McAvay G, Claus EB, Garrett P, Gottschalk $M$. et al. A multifactorial intervention to reduce the risk of falling among elderly people living in the community. $\mathrm{N}$ Engl J Med. 1994; 331:821-7.

10. Kallin K, Lundin-Olsson L, Jensen J, Nyberg L, Gustafson Y. Predisposing and precipitating factors for falls among older people in residential care. Public Health. 2002; 116:263-71.

11. Rubenstein LZ, Josepfson KR, Trueblood PR, Looy S. Effects of a group exercise program on strength, mobility, and falls among fall-prone elderly men. J Gerontol A Biol Sci Med Sci. 2000; 55:317-21.

12. Verhagen AP, Immink M, Van der Meulen A, Sita MA. The Efficacy of Tai chi chuan in older adults: a systematic review. Family Practice. 2004; 21:107-13.

13. Nolwalk M P, Prendergast JM, Bayles C M, Damico FJ, Colvin GC. A Randomized Trial of Exercise Programs among Older Individuals Living in Long-Term Care Facilities: The Falls FREE Program. J Am Geriatr Soc. 2001; 49:859-65.

14. Wang C, Collet JP, Lau J. The effect of Tai Chi on health outcomes in patients with chronic conditions: a systematic review. Arch Inter Med. 2004; 164:493-501.

15. Lin MR, Hwang HF, Wang YW, Chang SH, Wolf SL. Community-based tai chi and its effect on injurious falls, balance, gait and fear of falling in older people. Phys Ther. 2006; 86:1189-201.

16. Zeeuwe PE, Verhagen AP, Bierma-Zeinstra SM, van Rossum $E$. The effect of Tai Chi Chuan in reducing falls among elderly people: design of a randomized clinical trial in the Netherlands. BMC Geriatr. 2006; 30:6.

17. Kuei-Min Chen, Snyder M, Krichbaum K. Clinical use of taichi in elderly populations. Geriatric Nursing. 2001; 22:198200.

18. Lan C, Lai JS, Chen SY. Tai Chi Chuan. An ancient Wisdom on Exercise and Health Promotion. Sports Med. 2002; 32:217-22.

19. Wong AM, Lin YC, Chou SW, Tang FT, Wong PY. Coordination exercise and postural stability in elderly people: effect of Tai Chi Chuan. Arch Phis Med Rehabil. 2001; 82:608-12.

20. Hong Y, Li JX, Robinson PD. Balance control, flexibility, and cardiorespiratory fitness among older Tai Chi practitioners. Br J Sports Med. 2000; 34:29-34.

21. Li JX, Hong Y, Chan Km.Tai chi: Physiological characteristics and beneficial effects on health. Br J Sports Med. 2001; 35:148-9.

22. Wolf SL, Coogler $\mathrm{C}, \mathrm{Xu}$ T. Exploring the basis for Tai Chi Chuan as a therapeutic exercise approach. Arch Phys Med Rehabil. 1997; 78:886-92.

23. Kuramoto AM. Therapeutic benefits of Tai Chi exercise: research review. WMJ. 2006; 105:42-6.

24. Wolf SL, Barnhart HX, Kunter NG, McNeel E, Coogler C, Xu T. Atlanta FICSIT Group. Reducing Frailty and Falls in Older Persons: An Investigation of Tai Chi and Computerized Balance Training. J Am Geriatr Soc. 1996; 44:489-97.

25. Tsang WW, Wong VS, Fu SN, Hui-Chan CW. Tai Chi improves standing balance control under reduced or conflicting sensory conditions. Arch Phys Med Rehabil. 2004; 85:12937.

26. Verhagen AP, Immink M, van der Meulen A, Bierme-Zeinstra SM. The efficacy of Tai Chi Chuan in older adults: a systematic review. Fam Pract. 2004; 21:107-13.

27. Lan C, Lai JS, Chen SY, Wong MK. Tai Chi Chuan to improve muscular strength and endurance in elderly individuals: a pilot study. Arch Phys Med Rehabil. 2000; 81:604-7.

28. Xu D, Hong Y, Li J, Chan K. Effect of tai chi exercise on proprioception of ankle and knee joints in old people. Br J Sports Med. 2004; 38:50-4.

29. Wolf SL, Sattin RW, Kutner M, O'Grady M, Greenspan Al, Gregor RJ. Intense Tai Chi Exercise Training and Fall Occurrences in Older, Transitionally Frail Adults: A Randomized, Controlled Trial. J Am Geriatr Soc. 2003; 51:1693-701.

30. Deschamps A, Onifade C, Decamps A, Bourdel-Marchasson I. Health-related quality of life in frail institutionalized elderly: effects of a cognition-action intervention and Tai Chi. J Aging Phys Act. 2009; 17:236-48. 